\title{
Endothelium-specific loss of murine thrombomodulin disrupts the protein $C$ anticoagulant pathway and causes juvenile-onset thrombosis
}

\author{
Berend Isermann, ${ }^{1}$ Sara B. Hendrickson, ${ }^{1}$ Mark Zogg, ${ }^{1}$ Mark Wing, ${ }^{1}$ \\ Marjorie Cummiskey, ${ }^{2}$ Yaz Y. Kisanuki, ${ }^{3}$ Masashi Yanagisawa, ${ }^{3}$ \\ and Hartmut Weiler ${ }^{1,2}$

\begin{abstract}
${ }^{1}$ Blood Research Institute, The Blood Center of Southeastern Wisconsin, Milwaukee, Wisconsin, USA
${ }^{2}$ Medical College of Wisconsin, Milwaukee, Wisconsin, USA

${ }^{3}$ Department of Molecular Genetics, University of Texas Southwestern Medical Center, Dallas, Texas, USA

Address correspondence to: Hartmut Weiler, Blood Research Institute, The Blood Center

of Southeastern Wisconsin, PO Box 2178, Milwaukee, Wisconsin 53226, USA.
\end{abstract} \\ Phone: (414) 937-3813; Fax: (414) 937-6284; E-mail: hweiler@bcsew.edu.
}

Received for publication April 19, 2001, and accepted in revised form July 4, 2001.

\begin{abstract}
The thrombomodulin (TM) gene was ablated in mice in a cell type-restricted manner from vascular endothelium by Cre-recombinase-mediated excision controlled by the endothelial cell lineage-specific Tie2 promoter. Forty percent of mutant (TMLox-) mice display a distinct lethal embryonic phenotype not observed in completely TM-deficient embryos. The remaining $60 \%$ of TMLox mice survive beyond birth, but invariably succumb to a severe hypercoagulable state and massive thrombosis after 3 weeks, terminating in a lethal consumptive coagulopathy. The progression of thrombosis was age- and sex-dependent. Disruption of the TM/protein C pathway was not associated with a latent proinflammatory state. Disease onset and progression could be prevented by warfarin anticoagulation. These results show that in mice, loss of endothelial cell TM function causes spontaneous and fatal thrombosis in the arterial and venous circulation, resulting from unfettered activation of the coagulation system. The combination of complete disease penetrance, uniform disease onset at young age, large vessel thrombosis of the extremities and multiple organ systems, and consumptive coagulopathy as the disease end-point provides a unique mouse model of human thrombotic disease.
\end{abstract}

J. Clin. Invest. 108:537-546 (2001). DOI:10.1172/JCI200113077.

\section{Introduction}

The thrombomodulin (TM) protein C (PC) pathway provides a natural anticoagulant mechanism that inhibits the activity and excessive generation of the blood coagulation protease thrombin $(1,2)$. The association of thrombin and endothelial cell surface-associated TM inhibits the procoagulant activity of thrombin and redirects its substrate specificity toward the activation of PC and the inhibitor of fibrinolysis, TAFI (3). Activated PC also suppresses the elaboration of proinflammatory cytokines, improves the outcome of acute sepsis in human patients, and protects animals against the lethal consequences of experimental septicemia (4-8). Acquired or inherited deficiency of PC or protein $\mathrm{S}$ (PS) confers an increased risk of venous thrombosis (9). Congenital complete PC or PS deficiency causes purpura fulminans, a neonatal thrombohemorrhagic disorder that is fatal if left untreated $(10,11)$. Severe inherited deficiencies of TM have not been described in humans. The first TM gene mutation shown to result in reduced TM function has been identified only recently in patients suffering from myocardial infarction (12), and decreased plasma concentration of soluble TM correlates with an increased incidence of arteriosclerosis
(13). Other TM gene polymorphisms may be associated with augmented disease risk, but the evidence from these studies remains weak (14).

Gene targeting in mice showed that a complete loss of TM function is incompatible with early embryonic development (15). TM-null mice are invariably aborted before embryonic day 9.5 (E 9.5), precluding the analysis of TM function in adult mice. Subsequent studies revealed that development of TM-null mice proceeds normally beyond E 9.5 as long as TM expression is selectively maintained in trophoblast and/or parietal endoderm cells of the mouse placenta (16). This result established that the primary defect in TM-null embryos is not caused by the loss of TM from vascular endothelium, but resides in nonendothelial cells of the placenta. Despite the rescue from early lethality, TMnull mice nevertheless succumb to a lethal consumptive coagulopathy in utero between E 12.5 and E 16.5. These findings prompted us to investigate whether the selective depletion of the mouse TM gene from the vascular endothelial cell lineage might be compatible with embryonic development and enable studying the role of TM in the regulation of intravascular hemostasis in adult mice. We show that the ablation of TM in cells 
producing Cre-recombinase under the transcriptional control of the endothelial cell-specific tie2-promoter/enhancer indeed enables $60 \%$ of the mutant mice to survive beyond birth. The animals are viable, yet succumb at an early age to severe thrombosis that terminates in a fatal consumptive coagulopathy if left untreated. Unexpectedly, the remaining $40 \%$ of mutant mice succumb to a novel developmental defect not observed in completely TM-deficient mice.

\section{Methods}

Gene targeting and generation of transgenic animals. The structure of the gene-targeting vector is shown in Figure 1a. A polyadenylation signal derived from the mouse PGK-I ${ }^{\mathrm{b}}$ gene followed by a neomycin geneexpressing cassette was inserted into a unique SpeI site within the 3 -untranslated region of the mouse TM gene. LoxP sequences were inserted into the $5^{\prime}$-untranslated region adjacent to the translation initiation codon of the TM gene and at the 3 -end of the neomycin gene-expressing cassette.

Targeting of the TM-gene in D3 embryonic stem (ES) cells and selection of drug resistant ES cell clones was performed as described previously (17). Correctly targeted ES cell clones were identified by Southern blot hybridization of genomic DNA digested with NcoI and KpnI. Hybridization with a DNA probe external to the TM gene sequences contained in the targeting vector (Figure 1a, probe A) resulted in $3.75-\mathrm{kb}$ and $4.05-\mathrm{kb}$ signals for the wild-type TM allele ( $\left.\mathrm{TM}^{\mathrm{wt}}\right)$ and the correctly targeted TM allele (TM $\left.{ }^{\text {Lox-neo }}\right)$, respectively (Figure $\left.1 \mathrm{~b}\right)$. Mice heterozygous for the targeted mutation ( $\left.\mathrm{TM}^{\mathrm{wt} / \mathrm{Lox}-\mathrm{neo}}\right)$ were generated by blastocyst injection with targeted ES cells and breeding of the resulting germline chimeras to C57BL/ 6 females. $\mathrm{TM}^{\mathrm{wt} / \mathrm{Lox}-\mathrm{neo}}$ mice were then bred to transgenic mice expressing Cre-recombinase under the control of the tie2-promoter/enhancer (18), resulting in $\mathrm{TM}^{\mathrm{wt} / \mathrm{Lox}-}$ $\mathrm{Cr}^{\mathrm{tg}}$ mice. To maximize the ratio of Cre-recombinase product over its substrate (the conditional TM gene), we established a mouse strain that was heterozygous for the TM loss-of-function mutation (TM $\left.{ }^{\text {lacZ }}\right)(16,17)$ and at the same time carried the Cre-transgene ( $\left.\mathrm{TM}^{\mathrm{wt} / \mathrm{lacZ}} \mathrm{Cre}^{\mathrm{tg}}\right)$. $\mathrm{TM}^{\mathrm{wt} / \mathrm{lac} Z} \mathrm{Cre}^{\mathrm{tg}}$ and $\mathrm{TM}^{\mathrm{wt} / \mathrm{Lox}} \mathrm{Cre}^{\mathrm{tg}}$ mice were then bred to yield experimental mice (TMLox, genotype $\mathrm{TM}^{\mathrm{Lox} / \mathrm{lac} Z} \mathrm{Cr} \mathrm{e}^{\mathrm{tg}}$ ) and control mice (TMwt, sex-matched littermates, genotype $\left.\mathrm{TM}^{\mathrm{wt} / \mathrm{lac} Z} \mathrm{Cre} \mathrm{e}^{\mathrm{tg}}\right)$. Offspring were genotyped using genomic DNA isolated from a tail biopsy and employing multiplex PCR (Figure 1c). All animals were housed and experiments performed in the Animal Resource Center at the Medical College of Wisconsin, following standards and procedures approved by the local Animal Care and Use Committee.

In vivo $P C$ activation. Animals were injected with $20 \mu \mathrm{g}$ of human PC and $50 \mathrm{mU}$ of $\alpha$-thrombin (Enzyme Research Laboratories, South Bend, Indiana, USA) into the vena femoralis. Blood samples were collected after 10 minutes from the vena cava into $0.38 \%$ sodium citrate and $50 \mathrm{mM}$ benzamidine $\mathrm{HCl}$ (final concentration). Human PC/activated PC (APC) was captured from plasma samples using an antibody specific for human PC/APC (HAPC 1555; kind gift of G. Ferrell, Oklahoma City, Oklahoma, USA), and the activity of the captured human PC was determined using a chromogenic substrate (PCa; American Diagnostica, Greenwich, Connecticut, USA) (19). Background (TM-independent) APC formation was determined by adding thrombin and $\mathrm{PC}$ at concentrations corresponding to the final concentration achieved in vivo to TM-depleted mouse plasma. PBMCs were prepared by density gradient centrifugation (Nyco-Prep 1.077 A; Nycomed Pharmas, Torshov, Norway), followed by adherence to tissue culture dishes as described elsewhere (20). Adherent cells were greater than $95 \%$ monocytes as assessed by Giemsa staining. Peritoneal macrophages were isolated 4 days after intraperitoneal injection of Brewer's thioglycollate broth (4\%) (21). TM-dependent APC formation by mononuclear cells was established using a two-step assay for PC activation (22).

Gonadectomy. For removal of testes, a small incision in the anterior abdominal wall was made, the ductus deferens and adjacent blood vessels were ligated, testes removed, and the wound closed with a metal clip. Ovaries were removed through an incision over the lower back. The uterine tube, the ligaments attached to the ovary, and adjacent blood vessels were ligated, the ovaries removed, and the skin closed with a metal clip. Only a skin incision was made in sham-operated animals.

Warfarin anticoagulation. Warfarin [3-( $\alpha$-Acetonylbenzyl)-4-Hydroxy-Coumarin; Sigma Chemical Co., St. Louis, Missouri, USA] was added to the drinking water at a concentration of $2 \mu \mathrm{g} / \mathrm{ml}$ (freshly prepared three times a week). This dosage prolonged whole-blood clotting time from 6.8 to 24.9 minutes $(P<0.001)$ and reduced factor $\mathrm{X}$ activation by $73 \%(P<0.001)$, but caused hemorrhagic complications only in a minority of mice. Anticoagulation of experimental (TMLox) and control (TMwt) mice was initiated in mice less than 3 weeks old and continued for a period of 8 weeks. A placebo group received normal water only.

Analyses of blood coagulation, plasma cytokines, cross-linked tissue fibrin, and hematological parameters. Whole-blood clotting time was determined by monitoring the formation of a blood clot while stirring with a metal loop every 30 seconds for the first 10 minutes, and thereafter every 2 minutes up to 60 minutes. Blood samples for other assays were obtained from the inferior vena cava of anticoagulated mice $(8,000 \mathrm{U}$ heparin intraperitoneally, unfractionated porcine heparin, sodium-salt; Life Technologies Inc., Grand Island, New York, USA). Thrombinantithrombin complexes (TAT) and D-Dimer were determined using commercially available ELISA systems that recognized mouse TAT (23) and mouse D-Dimer (24), respectively (Enzygnost TAT micro; Behring Diagnostic Inc., Westwood, Massachusetts, USA, and Asserachrom D-Di; American Bioproducts Co., Parsippany, New Jersey, USA, respectively). Fibrinogen levels were measured by sandwich ELISA using a rabbit anti-human fibrinogen antibody (DAKO Corp., Carpinteria, California, 
Figure 1

(a) Targeted mutagenesis of the wild-type TM gene $\left(T M^{w t}\right)$ results in the insertion of a loxP site immediately $5^{\prime}$ of the translation initiation codon and $3^{\prime}$ of the neomycin gene-expression cassette (neo). TK, thymidine kinase expression cassette. (b) Correctly targeted ES cells were identified by Southern blot hybridization analysis with external probe A (see a). (c) Mouse DNA was analyzed by PCR using the sense primer $\mathrm{P}_{1}\left(5^{\prime}\right.$ aggttgtgatagaggctagctgctgtc $\left.-3^{\prime}\right)$ and the antisense primer $\mathrm{P}_{2}\left(5^{\prime}\right.$ - ggccctaaatccatactgctct $\left.-3^{\prime}\right)$, resulting in a 376-bp fragment for the TM wild-type allele, and the antisense primer $\mathrm{P}_{3}\left(5^{\prime}\right.$ - atgtgctgcaaggcgattaagttgg $\left.3^{\prime}\right)$, resulting in a 199-bp fragment for the $T M^{\text {lacz }}$ allele (primer $\mathrm{P}_{3}$ is derived from the $\mathrm{TM}^{\mathrm{lacz}}$ gene and is not shown in Figure 1a). The antisense primer $\mathrm{P}_{4}\left(5^{\prime}\right.$ - ggcccagtatgtctcaagatagcaatg $-3^{\prime}$ ) yielded a 408-bp fragment only after deletion of the endogenous TM gene by Cre-recombinase. Immunohistochemical detection of TM in the lung ( $d$ and $\mathbf{g}$ ), the brain (e and $\mathbf{h}$ ), and the placenta $(\mathbf{f}$ and $\mathbf{i})$ in TMwt $(\mathbf{d}-\mathbf{f})$ and TMLox $(\mathbf{g}-\mathbf{i})$ mice. Brown-black horseradish peroxidase reaction product identifies $T M$ antigen. In TMLox mice, TM is absent in almost all lung capillaries (g) and brain endothelial cells ( $\mathbf{h}$, arrows), whereas nonendothelial cells such as meninges (h, arrowheads), giant trophoblast and parietal endoderm cells $(\mathbf{i}$, arrowheads identify parietal endoderm) stain for TM. Arrows in $\mathbf{e}$ and $\mathbf{f}$ indicate meninges (top) and blood vessels. Original magnification is indicated at lower right.

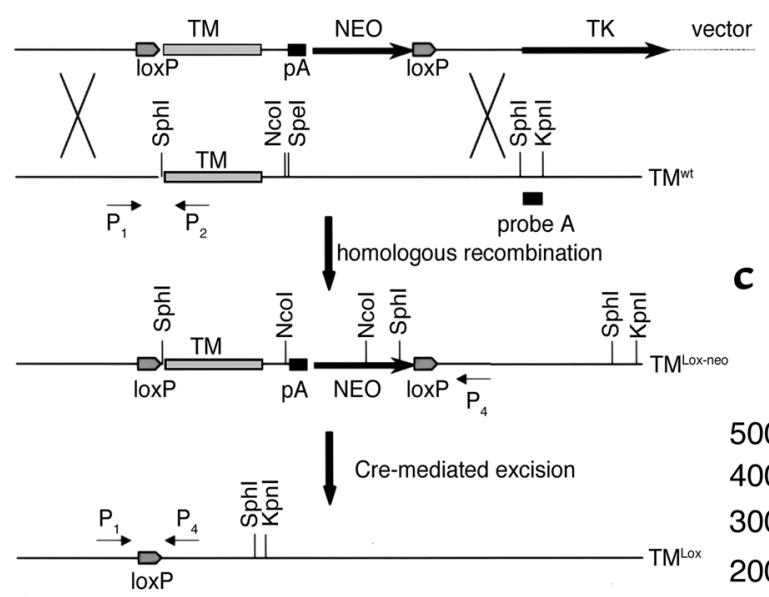

b
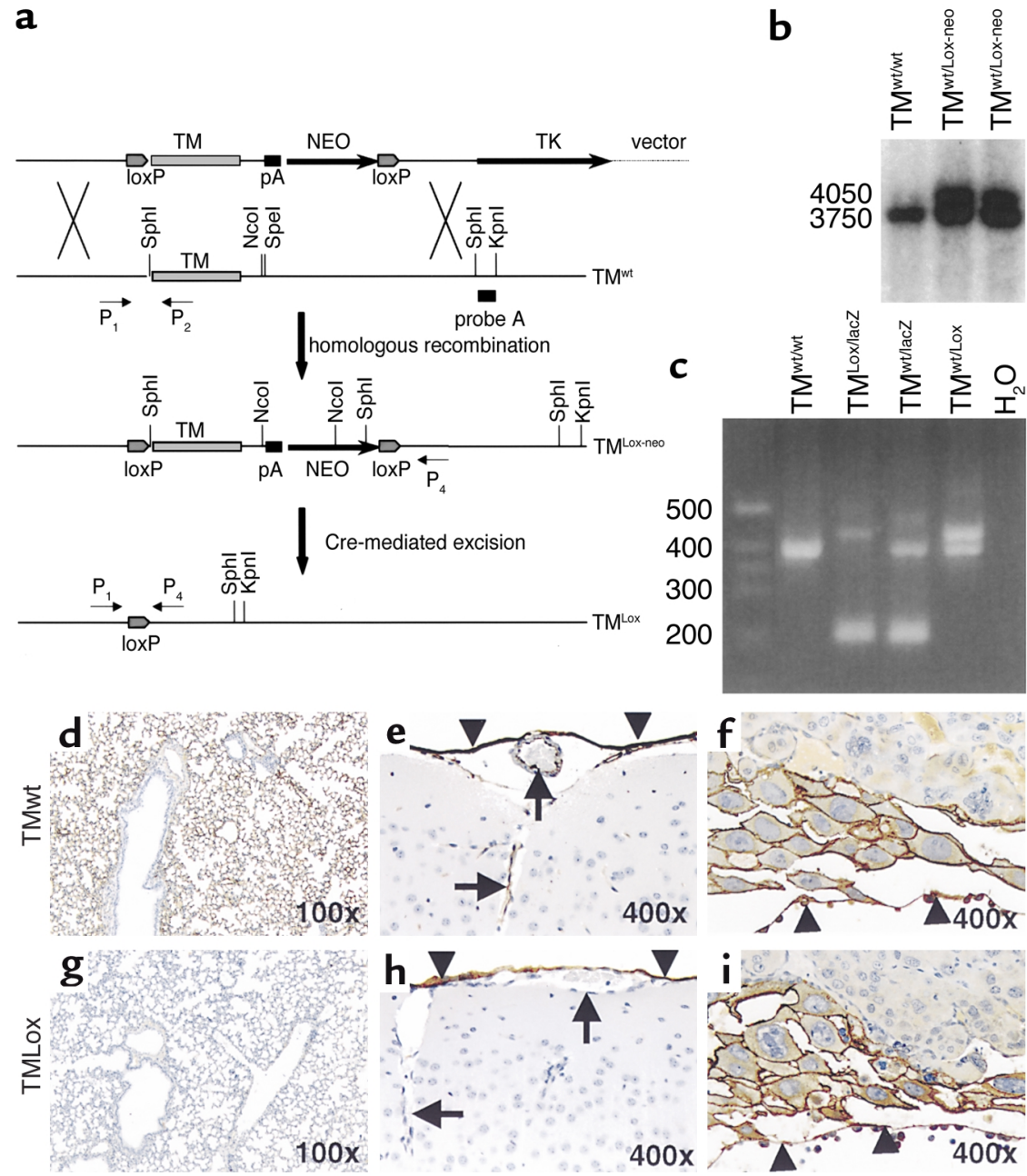

USA) and a peroxidase-conjugated goat anti-mouse fibrinogen antibody (Accurate International, Westbury, New York, USA). The assay was calibrated with purified mouse fibrinogen (a kind gift of M.W. Mosesson, Blood Research Institute, Milwaukee, Wisconsin, USA). Plasma IL-1 $\beta$ and IL- 6 were measured with mouse specific ELISA systems (R\&D Systems Inc., Minneapolis, Minnesota, USA). TM-antigen and cross-linked fibrin in tissue extracts were measured as described previously (16, $22)$. Megakaryocytes numbers in the spleen were determined by disruption of the spleen (60-count wire mesh; Sigma Chemical Co.) in RPMI-5\% FBS, centrifugation (400 $\mathrm{g}$ for 5 minutes), and incubation of resuspended cells in TRIS ammonium chloride $(0.75 \% \mathrm{wt} / \mathrm{vol}$, in TRIS [pH 7.2]) for 5 minutes at $37^{\circ} \mathrm{C}$. Cells were washed twice in RPMI-5\% FBS, transferred onto glass slides with a cytospin ( $150 \mathrm{~g}$ for 6 minutes), air dried, and stained for acetylcholinesterase (25). The number of megakaryocytes per slide was normalized to the total number of splenocytes per slide. Leukocyte and platelet numbers were counted manually.

Histology and immunohistochemistry. Tissues were fixed in $4 \%$ buffered paraformaldehyde, paraffin embedded, and processed for sectioning. Tissues containing bones were fixed and decalcified in CAL-EX (Fisher Scientific Co., Fair Lawn, New Jersey, USA). Immunohistochemical detection of TM with the TM-specific $\mathrm{mAb} 201 \mathrm{~B}$ and of fibrinogen with a polyclonal rabbit anti-human fibrinogen antibody (DAKO Corp.) was performed as described previously (16). Gomori's one-step trichrome stain was used for the visualization of interstitial collagen.

Statistical analyses. Results are shown as mean \pm SEM. Statview software (SAS Institute, Cary, North Carolina, USA) was used for all statistical analyses. The unpaired Student's $t$ test was used to compare values between TMLox mice and TMwt mice, except for the comparison of body weights, where the paired Student's $t$ test was used to compare sex-matched littermates. KaplanMeier curves and logrank test were employed to analyze the time course of disease manifestation and death.

\section{Results}

Generation of mice with endothelial-specific TM ablation. The DNA-targeting construct containing the loxP sites is shown in Figure 1a. Homologous recombination between the targeting construct and the endogenous TM gene resulted in the insertion of loxP sides flanking the TM gene and the neomycin expression 


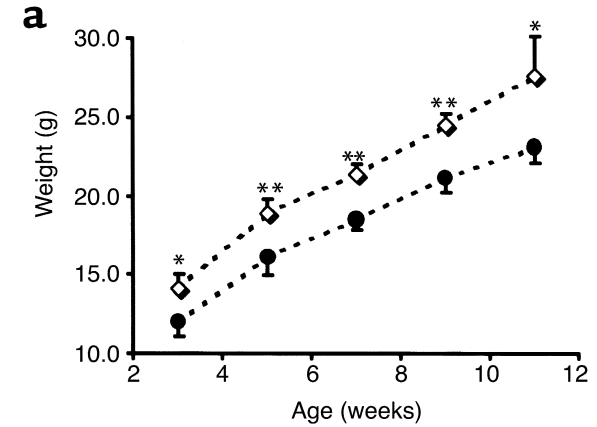

b

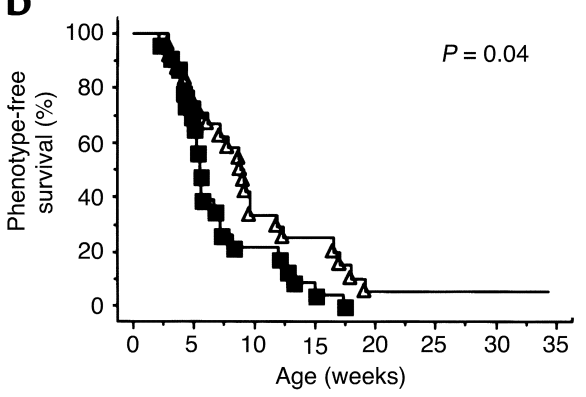

C

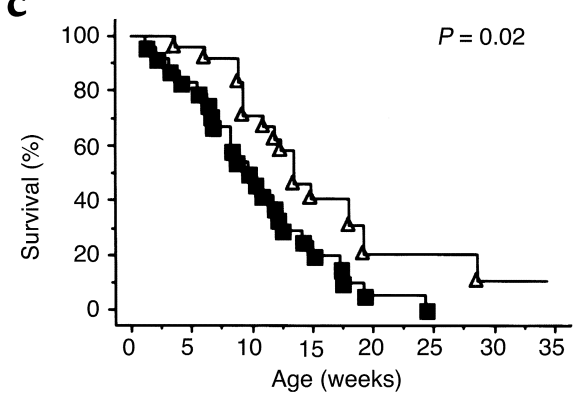

Figure 2

Disease progression and death in TMLox mice. Reduced body weight, phenotypical disease onset, and death in TMLox mice at young age. (a) Body weight in TMwt and TMLox littermates. The body weight of TMLox mice is reduced at weaning age and remains low thereafter. TMLox mice (excluding runt animals), filled circles; TMwt mice, open diamonds. Paired $t$ test; $n=27$; error bars $=$ SEM. ${ }^{*} P<0.05,{ }^{*} P<0.01$. Manifestation of phenotypes (b) and death (c) approaches $100 \%$ within 20 and 30 weeks, respectively, and occurs earlier in male than in female mice. Males, filled squares; $n=23$. Females, open triangles; $n=25$.

cassette (Figure 1a, TM ${ }^{\text {Lox-neo }}$ ). Two lines of mice with the correctly targeted $\mathrm{TM}^{\text {Lox-neo }}$ gene were established from two independently generated ES cell lines, and both mouse lines produced the same phenotype. Mice derived from only one line were used for the majority of subsequent experiments. Disruption of the TM gene in endothelial cells was achieved by crossing $\mathrm{TM}^{\mathrm{wt} / \mathrm{Lox}} \mathrm{Cre}^{\mathrm{tg}}$ and $\mathrm{TM}^{\mathrm{w} t} / \mathrm{lacz} C \mathrm{Cre}^{\mathrm{tg}}$ mice, yielding $\mathrm{TM}^{\mathrm{Lox} / \mathrm{lacZ}} \mathrm{Cre}^{\mathrm{tg}}$ ("TMLox") mice. This breeding strategy yielded also mice harboring the floxed TM gene $\left(\mathrm{TM}^{\mathrm{Lox}-\mathrm{neo}}\right)$ as well as the inactivated $\mathrm{TM}^{\text {lac }}$ allele, but lacking the Cre-transgene (TM ${ }^{\text {Lox-neo/lacZ }}$ ). $\mathrm{TM}^{\text {Lox-neo/lacZ }}$ mice developed normally and appeared healthy throughout adulthood, indicating that the $\mathrm{TM}^{\text {Lox-neo }}$ allele sustains sufficient and correctly reg- ulated TM expression during development and adulthood. The preservation of tissue-specific expression of TM was confirmed by immunohistochemistry (data not shown).

The Cre-Lox-dependent excision of TM in experimental TMLox mice $\left(\mathrm{TM}^{\mathrm{Lox} / \mathrm{lacz}} \mathrm{Cre}{ }^{\mathrm{tg}}\right)$ was confirmed by PCR as described in Methods (Figure 1c). Analyses of TM expression in tissue extracts obtained from TMLox mice and TM-expressing control mice (TMwt mice) revealed dramatically reduced, but not completely absent, TM levels (data not shown). In vivo PC activation of TMwt, TMLox, and heterozygous TM $\left(\mathrm{TM}^{\mathrm{wt} / \mathrm{lac} Z}\right)$ mice injected with human PC and $\alpha$ thrombin was reduced to $61 \%( \pm 30 \%)$ and $34 \%$ $( \pm 11 \%)$ in $\mathrm{TM}^{\mathrm{wt} / \mathrm{lac} Z}$ and TMLox mice, respectively, compared with TMwt mice. PBMCs and thioglycollate-stimulated peritoneal macrophages from TMwt, TMLox, and $\mathrm{TM}^{\mathrm{wt} / \mathrm{lacZ}}$ mice did not exhibit detectable PC activation. PBMCs lacked immunohistochemically detectable TM. The more sensitive $\beta$-galactosidase stain indicated TM expression in less than $0.1 \%$ of PBMCs derived from TM ${ }^{\mathrm{wt} / \mathrm{lacZ}}$ mice. Immunohistochemistry using a TM-specific antibody was used to determine whether the observed residual TM expression and in vivo PC activation was secondary to incomplete ablation of the TM gene in endothelial cells. TM expression was detected in less than $1 \%$ of lung and brain capillaries, but was not detected in any other small and large blood vessels in 3- and 8-week-old TMLox mice (Figure 1, g and h). Conversely, TM expression was readily detected in nonendothelial, TM-expressing tissue such as the meninges (Figure 1h), in testicular interstitium, in pericardium, and in parietal endoderm and trophoblast cells of E 9.5 TMLox mouse embryos (Figure 1i) indicating tissue-specific inactivation of the TM-gene. Yolk sac vasculature of $\mathrm{E} 9.5$ postcoitus (pc) embryos showed patchy TM-staining ( $50 \%$ of yolk sac vessels), indicating incomplete gene ablation in vascular endothelium at this stage.

Partial embryonic lethality of TMLox mice. Three weeks after birth, only approximately $60 \%$ of the expected number of TMLox mice was found (Table 1). Timed matings revealed a normal frequency of TMLox embryos at days 9.5 and $10.5 \mathrm{pc}$, but a reduced number of mutant embryos at days 14.5 and $16.5 \mathrm{pc}$ (Table 1). All TMLox embryos retrieved at day 9.5 pc were phenotypically normal, whereas $36 \%$ (5 of 14) of TMLox embryos retrieved at day 10.5 pc displayed embryonic pallor or placental thrombosis. The remaining $64 \%$ of TMLox embryos at day $10.5 \mathrm{pc}$ appeared normal, closely matching the frequency of TMLox mice at later developmental stages (days 14.5 and $16.5 \mathrm{pc}$ ) and at weaning age. Taken together, partial embryonic lethality affecting $40 \%$ of TMLox embryos occurs around day $10.5 \mathrm{pc}$, whereas the remaining TMLox embryos survive until genotyping age. Of note, hemorrhage within the embryo proper was not observed in TMLox embryos at any stage. 

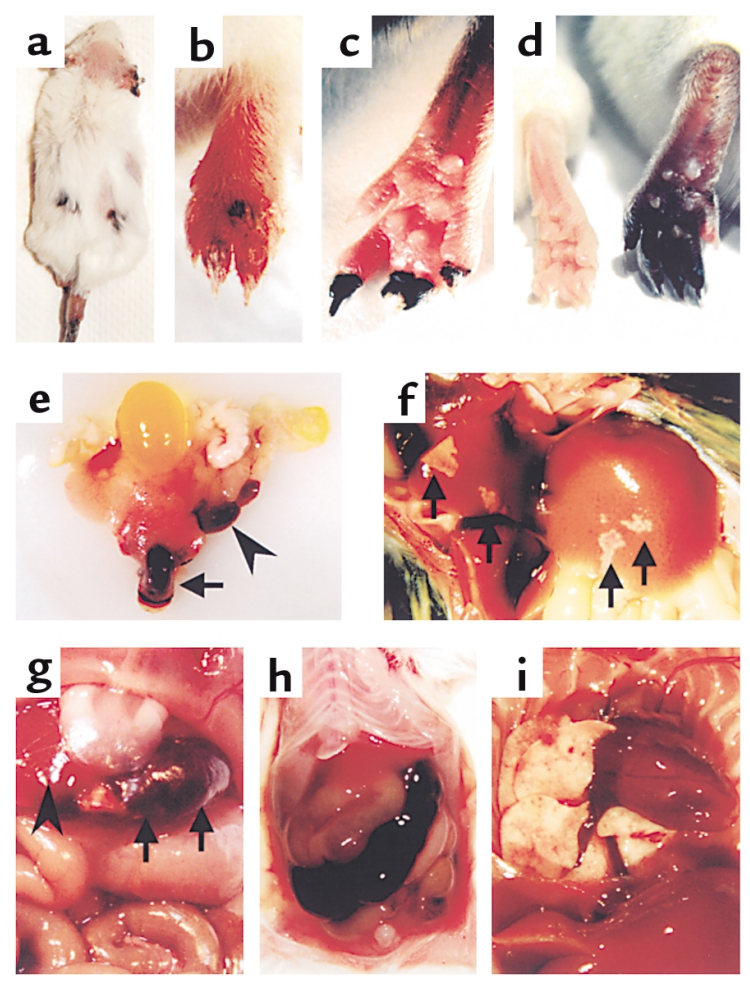

\section{Figure 3}

Thromboembolic disease in TMLox mice. TMLox mice develop hemorrhagic lesions and organ necrosis and succumb to consumptive coagulopathy. (a) Typical necrotic lesion of the skin, the ears, and the tail in TMLox mice. Early and small hemorrhagic skin lesions (b) subsequently progress into larger necrotic lesions (c and d; left foot in d: TMwt littermate). (e) Macroscopic specimen of a mouse with priapism: hemorrhagic necrosis of the penis (arrow) and testis (arrowhead). ( $\mathbf{f}$ and $\mathbf{g}$ ) Multiple necrosis of the liver ( $\mathbf{f}$, arrows) and swollen, hemorrhagic liver lobe (g, arrows) in TMLox mice (arrowhead in g: unaffected liver tissue). (h) Gastrointestinal bleeding at the disease end-stage in a TMLox mouse. (i) Multiple hemorrhagic lesions in the lung of a TMLox mouse.

Spontaneous thrombosis in TMLox mice. Born TMLox mice appeared generally healthy during the first postnatal weeks, except for a reduced body weight. At weaning age, $14 \%$ of TMLox mice were markedly smaller (runt), and in the remaining $86 \%$ of TMLox mice, the body weight (Figure $2 \mathrm{a}$ ) and weight gain were markedly reduced over an 8 -week period.

Between ages 3 and 20 weeks, 198 of 199 TMLox mice developed a macroscopically visible phenotype, or died spontaneously (Figure $2 \mathrm{~b}$ ). Study end points determining a "phenotype onset" were the occurrence of death secondary to consumptive coagulopathy or progressive thrombosis, hemorrhage/ necrosis of individual digits, extremities, skin, ears, tongue, or priapism, but excluded tail necrosis, as the latter was frequently triggered by tail cutting. Early skin lesion consisted of small hemorrhagic lesion (Figure 3b), which subsequently increased in size and became necrotic (Figure 3, a and c). Necrosis of distal phalanges was frequently associated with severe edema- tous swelling (Figure 3d). Skin lesions occurred on the extremities (tail, $77.9 \%$ of TMLox mice; hind limbs, $57.4 \%$ ), the perineal region (18.9\%), the abdominal skin (15.8\%), the ears (6.7\%), and at other sites (7.2\%). TMLox mice died either spontaneously $(37.2 \%)$ or were sacrificed $(57.1 \%)$ in the case of advanced disease. The overall survival of male mice was shorter than that of female mice (Figure 2c), and all mice surviving the observation period of 35 weeks were females (5.7\%). A sex difference was also apparent in the disease onset, as male mice developed a phenotype at a younger age than female mice (Figure $2 b$ ).

Upon autopsy and histological examination, liver lesions were prevalent in $35.9 \%$ of TMLox mice and presented either as liver infarcts $(26.9 \%$, Figure $3 \mathrm{f})$ or as hemorrhagic swelling of entire liver lobes, reminiscent of the Budd Chiari syndrome (9.0 \%, Figure $3 \mathrm{~g}$ ). Subcutaneous hemorrhage was found in $67 \%$ of TMLox mice. Other sites of hemorrhage included the gastrointestinal tract $(8 \%$, Figure $3 \mathrm{~h})$ and the lung $(10 \%$, Figure 3i). Hematuria was occasionally (1\%) observed in TMLox mice. Priapism (8\% of male mice), consistent with occlusion of efferent venous blood vessels, was repeatedly observed and was in some cases associated with necrosis of a testis (Figure 3e). In one mouse displaying a sudden onset of severe gait ataxia, the cerebellum was replaced with a liquid-filled cyst.

Histological examination confirmed multiple venous and arterial thrombi in small-and large-caliber blood vessels. Fibrin-rich blood clots with few cellular components and organized, frequently recanalized cellular thrombi rich in collagen and fibrinogen were present at multiple sites (Figure 4, a and b). Extravascular fibrinogen deposition was increased in lungs of TMLox mice at the earliest time point analyzed (3 weeks). The increased fibrinogen deposition in the lung was associated with an increase of extracellular matrix and a disruption of the tissue architecture, resulting in distention of terminal airways and alveoli, destruction of alveolar septa and, in severe cases, formation of bullae (Figure 4c). The hearts of TMLox mice were significantly enlarged at ages 5 and 8 weeks (Figure 5a), with

\section{Table 1}

Frequency of TMLox mice found at various gestational stages (days pc) and at weaning age ( 3 weeks)

Total number of mice

TMLox mice

Day 9.5

Day 10.5

Day 14.5

Day 16.5

3 weeks

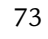

58

43

36

373

21

$14^{\mathrm{A}}$

6

5

$41^{\mathrm{B}}$

Numbers in parentheses reflect the expected frequency of TMLox mice based on the Mendelian frequency (3/16). AFive of the 14 TMLox embryos found at day 10.5 pc were abnormal, e.g., showed signs of embryonic pallor or placental thrombosis. ${ }^{\text {TThe frequency of TMLox mice found at weaning age was }}$ significantly reduced compared with the expected frequency of TMLox mice $\left(\chi^{2}=8.9 ; P<0.01\right)$. 

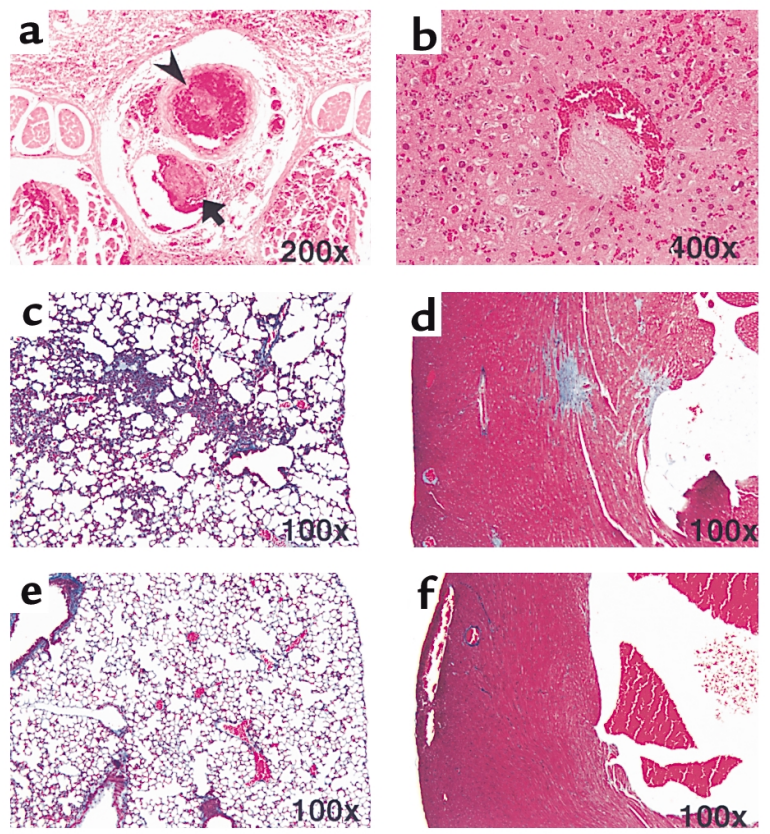

marked hypertrophy of the right ventricular wall (Figure $5 \mathrm{c}$ ). These findings are consistent with increased resistance in the pulmonary vascular bed as a consequence of recurrent embolic and/or vascular lung injury and subsequent pulmonary hypertension. In addition, increased tissue fibrin levels (data not shown) and multiple foci of myocardial fibrosis (Figure 4d) were apparent in 8-week-old TMLox mice, indicating thrombosis of cardiac blood vessels. Of note, no thrombus formation was observed in cardiac atria, which has been described in factor $V$ Leiden mice (26) and mice deficient in PC (27-29). Fibrin deposition, as determined by immunohistochemistry and Western blot analysis, was minimal in the brain of TMLox mice, whereas tissue fibrin levels in the lung and heart were increased (at age 3 and 8 weeks; data not shown).

Coagulation activation in TMLox mice. Three-week-old TMLox mice without visible lesions demonstrated increased TAT and D-Dimer levels, and augmented consumption of fibrinogen and platelets compared with TMwt littermates (Table 2). In 5- to 8-week-old TMLox mice, TAT and D-Dimer levels remained high or increased even further, consistent with the progressive severity of thrombotic complications. Conversely, fibrinogen levels increased and platelet numbers normal-

\section{Figure 5}

Cardiomegaly in TMLox mice. Hearts of TMLox mice were enlarged compared with hearts from TMwt mice. (a) The weights of whole hearts from TMLox and TMwt mice at age 3, 5, and 8 weeks, corrected for the body weight. Hearts tended to be larger at age 3 weeks and were significantly enlarged at 5 and 8 weeks in TMLox mice. (b and c) H\&E-stained histological sections obtained at comparative levels from hearts of TMwt (b) and TMLox (c) mice. The heart of the TMLox mice is overall enlarged with a striking hypertrophy of the right ventricular wall (arrowheads). Original magnification, $\times 20$.

\section{Figure 4}

Histopathological analyses of TMLox mice. Histological analyses revealed multiple pathological findings in TMLox mice. (a) Intravascular thrombi in a peripheral vein (arrow) and the adjacent artery (arrowhead); hematoxylin and eosin (H\&E) stain. (b) Intravascular thrombus in the liver, partially occluding a liver vein; H\&E stain. (c and e) Trichrome stain of lung sections from TMLox (c) and TMwt (e) mice. Increased extracellular matrix content in the lung interstitium is associated with a marked destruction of the lung architecture and a loss of alveolar septa. (d and $\mathbf{f}$ ) Trichrome stain of heart sections from TMLox (d) and TMwt (f) mice; multiple sites of myocardial fibrosis in the TMLox heart. Original magnification is indicated at lower right.

ized in older mice, indicating partial or complete compensation for consumption of fibrinogen and platelets. Consistent with compensatory platelet biosynthesis, the spleen weight (TMwt: $107.1 \pm 26.7 \mathrm{mg}$; TMLox $283.0 \pm 145.4 \mathrm{mg} ; P<0.001)$ and number of megakaryocytes per splenocytes (TMwt: $38 \pm 5.6 / 10^{5}$ splenocytes; TMLox $118 \pm 14.0 / 10^{5}$ splenocytes; $P=0.02$ ) were increased in 8-week-old, but not in 3-week-old, TMLox mice. Leukocyte numbers and hematocrit were identical between TMLox and TMwt mice at all ages. Evaluation of terminally diseased TMLox mice $(n=13)$ revealed a significantly prolonged whole-blood clotting time (TMwt: $6.5 \pm 0.9 \mathrm{~min}$, TMLox $37.4 \pm 6.2 \mathrm{~min}$; $P<0.001)$, and blood samples of six TMLox mice failed to form a blood clot within 60 minutes, suggesting consumptive coagulopathy as the disease end point. IL- $1 \beta$ and IL-6 levels were normal in 3-week-old TMLox mice compared with TMwt littermates, whereas in older TMLox mice with widespread organ damage IL-6 (but not IL-1 $\beta$ ), values were elevated (data not shown).

Sex hormones modify the disease progression in TMLox mice. To evaluate the contribution of sex hormones to the differential disease onset and progression in male and female animals, TMLox and control mice were

a

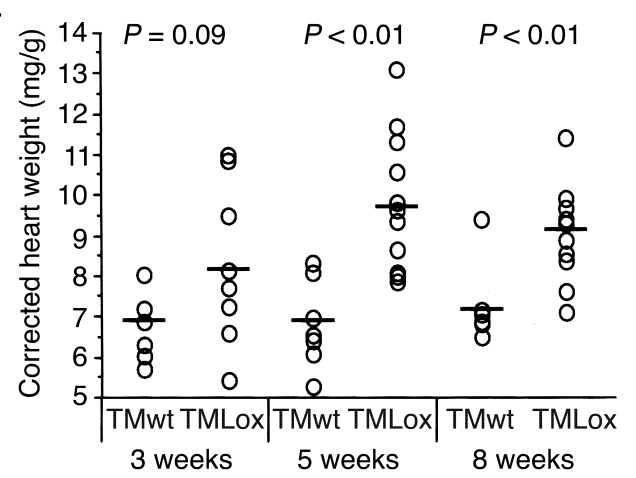

b
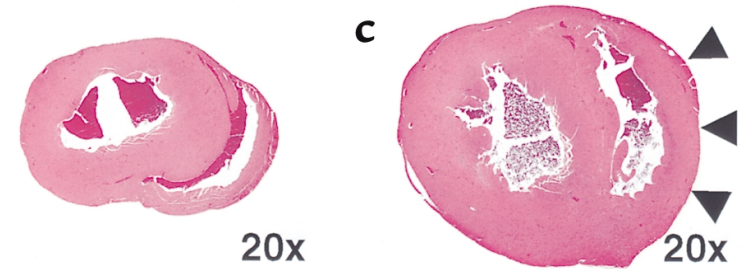
a

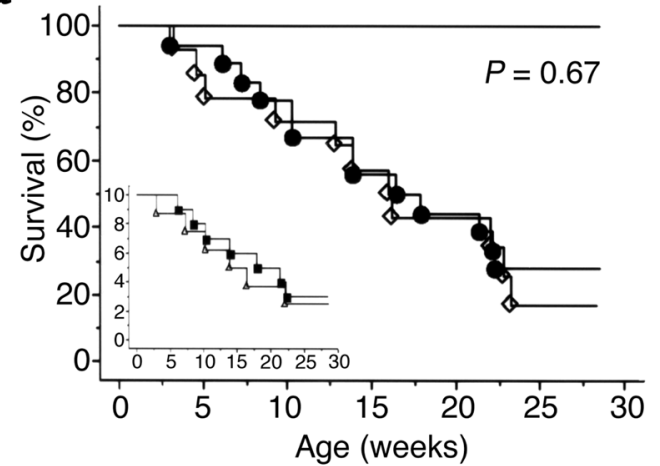

C

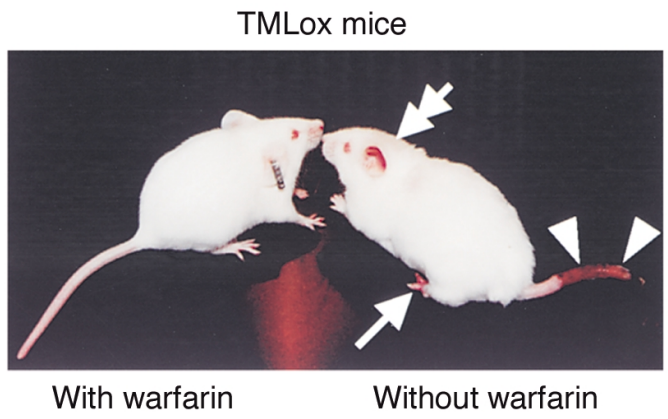

b

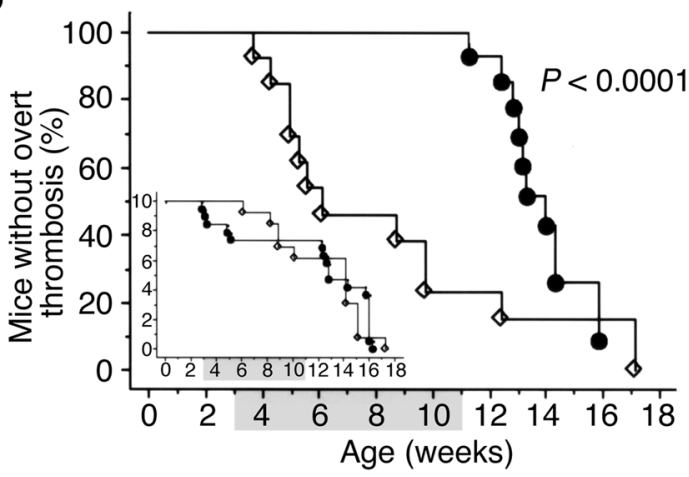

d

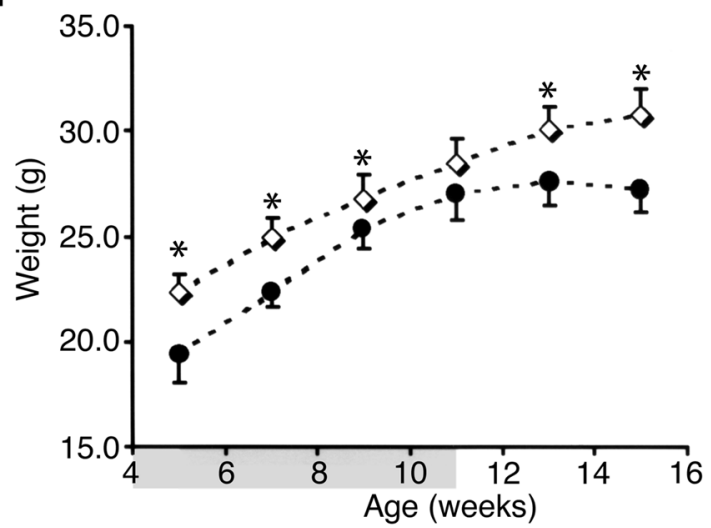

Figure 6

Effect of gonadectomy and anticoagulation on the disease progression and death in TMLox mice. (a) Gonadectomy before age 3 weeks did not prevent disease onset or death but reversed the sex-specific disease progression (inset). Gonadectomized TMLox mice, filled circles $(n=$ 18); sham-operated TMLox mice, open diamonds $(n=14)$. Inset: gonadectomized males, filled squares $(n=10)$; gonadectomized females, open triangles $(n=8)$. (b and $\mathbf{c})$ Warfarin treatment effectively prevents the overt thrombosis over an 8-week period (b, shaded area: warfarin treatment period), whereas typical lesions occur in mice from the placebo group (c, right; arrow: autoamputation of toes; arrowheads: hemorrhagic tail necrosis; double arrow: necrosis of the ear). The phenotype onset ("overt thrombosis") does not included warfarin-induced hemorrhage. After termination of warfarin treatment at age 11 weeks, mice rapidly succumb to the prothrombotic challenge. The overall survival of warfarin-treated TMLox mice is not improved secondary to warfarin-induced hemorrhagic side effects (inset). Warfarin-treated TMLox mice, filled circles $(n=18)$; water-treated (placebo-treated) TMLox mice, open diamonds $(n=14)$. (d) During the warfarin treatment period, the body weight of TMLox mice normalizes, and after 8 weeks of anticoagulation, the weight difference between TMLox mice and TMwt littermates (age 11 weeks) loses significance. Yet mice again fail to thrive once treatment is terminated. TMLox mice, filled circles; TMwt mice, open diamonds $(n=12)$. Paired $t$-test; ${ }^{*} P<0.05$. Shaded area: warfarin treatment period.

gonadectomized at age 18-19 days. Overall, the removal of gonads did not delay the manifestation of the phenotype or death (Figure 6a). Nevertheless, gonadectomy abolished the gender-specific differences in disease onset and mortality. On average, male TMLox mice succumbed at an older age to the consumptive coagulopathy than did female TMLox mice (Figure 6a, inset). This reversal of the disease course was not apparent in sham-operated TMLox animals.

Anticoagulation with warfarin prevents disease onset in TMLox mice. Oral anticoagulation of TMLox mice with warfarin between ages 3 and 11 weeks completely prevented the onset of overt thrombosis. Warfarin-treated TMLox mice did not develop hemorrhagic or necrotic skin lesion (Figure 6, b and c), and the internal organs appeared normal upon autopsy except for minor liver lesions in some animals (two of four TMLox mice ana- lyzed at the end of the treatment period). Total body weight (Figure 6d) and spleen weight (TMwt: $107.1 \pm 26.7 \mathrm{mg}$; anticoagulated TMLox $125.0 \pm 25.0 \mathrm{mg}$; $P=0.54$ ) normalized during the treatment. Nevertheless, warfarin treatment failed to improve overall survival in TMLox mice secondary to lethal cerebral or intrathoracic hemorrhage (Figure $6 \mathrm{~b}$, inset). The fatal hemorrhages in anticoagulated animals were likely a direct consequence of bleeding complications associated with warfarin treatment, as (a) they occurred at an identical rate in warfarin-treated TMwt and TMLox mice, and (b) the tissue sites of hemorrhage were similar in warfarintreated TMwt and TMLox mice, but distinct from the bleeding pattern observed in untreated TMLox mice with consumptive coagulopathy. Of note, initiation of warfarin therapy was not associated with appearance of purpuric skin lesions in TMLox or TMwt mice. 
Table 2

Blood coagulation parameters in control (TMwt) and experimental (TMLox) littermates

\begin{tabular}{lcccccc}
\hline Age (weeks) & \multicolumn{2}{c}{3} & & 5 & & 8 \\
Group & TMwt & TMLox & TMwt & TMLox & TMwt & TMLox \\
TAT & $3.0 \pm 0.2$ & $6.8 \pm 1.0^{\mathrm{A}}$ & $2.8 \pm 0.3$ & $5.8 \pm 0.8^{\mathrm{A}}$ & $3.2 \pm 0.1$ & $10.5 \pm 2.2^{\mathrm{A}}$ \\
D-Dimer & $27 \pm 8$ & $132 \pm 39^{\mathrm{B}}$ & $42 \pm 4$ & $199 \pm 59^{\mathrm{B}}$ & $15 \pm 2$ & $345 \pm 79^{\mathrm{A}}$ \\
Fibrinogen & $2.5 \pm 0.1$ & $1.3 \pm 0.2^{\mathrm{A}}$ & $2.2 \pm 0.1$ & $1.8 \pm 0.2$ & $2.6 \pm 0.1$ & $1.9 \pm 0.2^{\mathrm{A}}$ \\
Platelets & $1.2 \pm 0.05$ & $0.8 \pm 0.08^{\mathrm{B}}$ & $1.2 \pm 0.7$ & $1.1 \pm 0.05$ & $1.0 \pm 0.08$ & $1.0 \pm 0.08$ \\
\hline
\end{tabular}

Increased plasma levels of TAT (apparent micrograms per liter) and D-Dimer (apparent nanograms per milliliter), and decreased levels of plasma fibrinogen (milligrams per milliliter) in TMLox mice at age 3,5 , and 8 weeks. Platelets $\left(10^{6} / \mu \mathrm{l}\right)$ are reduced in TMLox mice age 3 weeks but not in older TMLox mice. Mean $\pm \mathrm{SEM} ; n=10$ for each group. ${ }^{\mathrm{A}} P<0.01 ;{ }^{\mathrm{B}} P<0.05$.

\section{Discussion}

The present results demonstrate that the tissuerestricted depletion of the TM gene in the endothelial cell lineage, as defined by tie 2 expression, is compatible with early embryonic growth and morphogenesis beyond the critical developmental block encountered by mice with complete TM deficiency. Nevertheless, $40 \%$ of the animals lacking endothelial TM die in utero around day $10.5 \mathrm{pc}$, whereas the remaining animals are born alive and survive the postnatal period. The reduction of TM in vascular endothelium nevertheless results in a spontaneous and progressive coagulopathy at young age. Onset and progression of overt disease occur in the absence of pronounced inflammatory changes and can be entirely prevented by oral anticoagulation therapy, indicating that excessive coagulation activation and ensuing thrombosis are the predominant underlying mechanism of disease caused by the depletion of TM from vascular endothelium.

TM-inactivation restricted by tie 2 promoter-dependent expression of Cre-recombinase bypassed the early midgestational developmental block encountered by completely TM-deficient embryos, but about $40 \%$ of TMLox embryos died at a later stage. The rescue of TMLox embryos from early midgestational lethality corroborates earlier results identifying nonendothelial placenta cells as the critical tissue sites requiring TM expression during development (16). TM-null tetraploid embryos, in which TM expression is restricted to the placenta, are rescued from early lethality, but succumb to intrauterine hemorrhage at a later stage. The partial embryonic lethality of TMLox embryos differs in two aspects from the intrauterine hemorrhage observed in TM-nulltetraploid embryos (16): First, TM-null tetraploid embryos die between days 12.5 and $16.5 \mathrm{pc}$, whereas the partial embryonic lethality of TMLox embryos occurs around day 10.5 pc. Second, TM-null tetraploid embryos succumb to a characteristic hemorrhage within the embryo proper that was not observed in any of the TMLox embryos at day 10.5 pc or later stages. These differences indicate that the partial embryonic lethality of TMLox embryos is the consequence of a distinct defect not observed in TM-nulltetraploid embryos. Given that the complete absence of TM from all cells except nonendothelial placental tissues is compatible with normal embryonic development (16), the defect causing partial embryonic lethality in TMLox mice must reside in the placenta. Expression of tie 2 in extraembryonic tissues has indeed been demonstrated in mice (30) and in humans $(31,32)$. In the latter, the endovascular trophoblast, a highly specialized trophoblast subpopulation invading and breaching maternal blood vessels, has been identified as a tie2-expressing cell population (31). Defects in the endovascular trophoblast are thought to impair pregnancy outcome in humans, e.g., in preeclampsia (33). Experiments have been initiated to investigate whether tie2-dependent TM inactivation similarly occurs in mouse endovascular trophoblast cells of TMLox embryos, and to determine the mechanism leading to catastrophic placental failure. That only some, but not all, TMLox mice succumb to this defect suggests that excision of the conditional TM allele in tie2-expressing trophoblast might be incomplete, similar to the highly efficient, but incomplete (87-99\%), deletion of floxed genes reported by others (34). Indeed, residual TM expression was also observed in embryonic blood vessel endothelium of the yolk sac, as well as in a small number of brain and lung capillaries. Spurious TM expression in endothelial cells, together with the sustained expression in nonendothelial cells, likely contributes to some extent to the substantial in vivo PC activation remaining in thrombin-challenged TMLox mice. The latter could explain not only the much better survival of TMLox animals, compared with PC-knockout mice (27), but also the absence of intrauterine hemorrhage observed in completely TM-deficient embryos (16). On the other hand, TM expression by mononuclear cells is unlikely to result in significant generation of activated PC in wild-type or mutant mice, as (a) TM gene expression was observed in less than $0.1 \%$ of mononuclear cells, and (b) cell preparations enriched for monocytes did not result in detectable PC activation. Animals bypassing the developmental impediment at day 10.5 pc are born healthy and lack a visible thrombotic disease until age 2-3 weeks. Nevertheless, virtually all of the mutant mice then develop a severe thrombosis, terminating in a lethal consumptive coagulopathy. The fate of TMLox mice resembles the catastrophic consequences of a disrupted TM/PC pathway in humans with PC or PS deficiency (11). The hall- 
mark of PC and PS deficiencies in humans are thrombotic occlusion of large peripheral veins and microvascular dermal thrombosis $(11,35,36)$. Other tissues, such as the liver, lung, and male genitals, are beginning to emerge as sides of primary thrombosis in these patients (37-41). The high frequencies of priapism and thrombotic liver and lung injury in TMLox mice, including such catastrophic sequelae as Budd Chiari syndrome, emphysema, and pulmonary hypertension, demonstrate that these manifestations are a direct consequence of a disrupted TM/PC pathway. The disease progression in TMLox mice is characterized by a transition from a markedly prethrombotic state into overt thrombosis of progressive severity. Elevated TAT and D-Dimer plasma levels and consumption of fibrinogen and platelets demonstrate the presence of a hypercoagulable state already at age 3 weeks. Within a short time thereafter (5 weeks in males, 9 weeks in females), $80 \%$ of mice develop overt thrombotic disease. Cardiac hypertrophy is already apparent in 3-week-old TMLox mice, indicating preexisting increased vascular resistance in the pulmonary circulation.

The coincidence of overt thrombotic lesions with sexual maturation, the gender differences in the disease evolution, and the age- and gender-dependent differences of the hemostatic system in humans (42-45) prompted us to investigate the effects of gonadal hormones on the onset and progression of phenotypically overt thrombosis. The unaltered disease course after gonadectomy of 18- to 19-day-old TMLox mice demonstrates that the sexual maturation and the associated hormonal changes do not trigger the transition from a severe prethrombotic or hypercoagulable state to overt thrombosis. Interestingly, gonadectomy eliminated the survival advantage of female mice, showing that gender-specific differences modulate disease progression through an undefined mechanism.

The efficiency of the warfarin treatment, together with the lack of evidence for excessive production of proinflammatory cytokines at early disease stages, implies that the underlying disease mechanism is almost exclusively caused by increased coagulation system activity. The prethrombotic phenotype in TMLox mice spontaneously progresses into a lethal consumptive coagulopathy in the absence of the complex cytokine changes observed in sepsis. Elevated IL-6 levels were observed only in older TMLox animals and might be related to excessive and widespread organ damage in these animals. Therefore, disruption of the TM/PC pathway per se is not sufficient to elicit alterations of proinflammatory cytokines, suggesting that the TM/PC pathway has no or only a minor role in controlling baseline levels of inflammatory cytokines.

Disruption of the TM/PC pathway in mice at the level of either TM or PC function elicits qualitatively distinct consequences that might reflect disparate physiological roles of individual pathway components. For example, atrial blood clots were observed only in mice lacking PC
(27-29) or carrying the factor V Leiden mutation (26), but not in TM-null tetraploid (16) or TMLox mice. Furthermore, both PC-deficient $(27,28)$ and factor V Leiden (26) mice displayed markedly increased fibrin deposition and intracerebral hemorrhage, whereas in mice with an impaired TM function, cerebral fibrin depositions and destruction of cerebral tissue are minor or absent $(16,22)$. Such distinct consequences of TM deficiency on the one hand, and PC deficiency or activated PC resistance on the other hand could be related to the preserved PC-independent anticoagulant function of TM (46) and/or the TAFI-dependent differences in the fibrinolytic system activity (3).

Mice with an endothelial specific disruption of the TM gene provide a unique mouse model for severe spontaneous thrombosis secondary to excess activation of the coagulation system that is caused by a single gene defect. The initial viability of mutant mice, the particular time course of disease onset at young age, the almost complete penetrance of overt thrombosis, involvement of the microvasculature as well as large venous and arterial vessels, and fatal bleeding due to coagulation factor consumption as the disease end point are unique features of this animal thrombosis model, setting it apart from previously described mice with genetically inactivated anticoagulant mechanisms $(22,26,27,47,48)$. These mice provide insight into the physiological role and importance of endothelial TM in vivo and constitute a valuable animal model for the evaluation of new therapeutic strategies for the treatment of inherited and acquired deficiencies of the $\mathrm{TM} / \mathrm{PC}$ pathway and, more generally, for establishing the efficacy and safety of new anticoagulant therapies.

\section{Acknowledgments}

We thank D. Beeler (Massachusetts Institute for Technology) for technical support in vector construction, and S. J. Kennel (Oak Ridge National Laboratory) for providing anti-mouse TM antibodies. This work was supported by NIH grant HL-60655, by a grant-in-aid from the American Heart Association (H. Weiler), and by a grant of the Deutsche Forschungsgemeinschaft (IS 67/1-2) to B. Isermann.

\footnotetext{
1. Esmon, C.T. 1987. The regulation of natural anticoagulant pathways. Science. 235:1348-1352.

2. Esmon, C.T. 2000. Regulation of blood coagulation. Biochim. Biophys. Acta. 1477:349-360

3. Bajzar, L., Morser, J., and Nesheim, M. 1996. TAFI, or plasma procarboxypeptidase B, couples the coagulation and fibrinolytic cascades through the thrombin-thrombomodulin complex. J. Biol. Chem. 271:16603-16608.

4. Bernard, G.R., et al. 2001. Efficacy and safety of recombinant human activated protein C for severe sepsis. N. Engl. J. Med. 334:699-709.

5. Taylor, F.B., Jr., et al. 1987. Protein C prevents the coagulopathic and lethal effects of Escherichia coli infusion in the baboon. J. Clin. Invest. 79:918-925.

6. Taylor, F., et al. 1991. C4b-binding protein exacerbates the host response to Escherichia coli. Blood. 78:357-363.

7. Hartman, D., Bernard, G.R., Helterbrand, J.D., Yan, S.B., and Fisher, C.J. 1998. Recombinant human activated protein C (rhAPC) improves coagulation abnormalities associated with severe sepsis. Intensive Care Med. 24:S77.

8. Esmon, C.T., et al. 1999. Regulation and functions of the protein C anti-
} coagulant pathway. Haematologica. 84:363-368. 
9. Stefano, D.V., Finazzi, G., and Mannucci, P.M. 1996. Inherited thrombophilia: pathogenesis, clinical syndromes, and management. Blood. 87:3531-3544

10. Marciniak, E., Wilson, H.D., and Marlar, R.A. 1985. Neonatal purpura fulminans: a genetic disorder related to the absence of protein $\mathrm{C}$ in blood. Blood. 65:15-20.

11. Marlar, R.A., and Neumann, A. 1990. Neonatal purpura fulminans due to homozygous protein $\mathrm{C}$ or protein $\mathrm{S}$ deficiencies. Semin. Thromb. Hemost. 16:299-309.

12. Kunz, G., et al. 2000. Identification and characterization of a thrombomodulin gene mutation coding for an elongated protein with reduced expression in a kindred with myocardial infarction. Blood. 95:569-576.

13. Salomaa, V., et al. 1999. Soluble thrombomodulin as a predictor of incident coronary heart disease and symptomless carotid artery atherosclerosis in the Atherosclerosis Risk in Communities (ARIC) Study: a casecohort study. Lancet. 353:1729-1734.

14. Ohlin, A.K., Norlund, L., and Marlar, R.A. 1997. Thrombomodulin gene variations and thromboembolic disease. Thromb. Haemost. 78:396-400.

15. Healy, A.M., Rayburn, H.B., Rosenberg, R.D., and Weiler, H. 1995. Absence of the blood-clotting regulator thrombomodulin causes embryonic lethality in mice before development of a functional cardiovascular system. Proc. Natl. Acad. Sci. USA. 92:850-854.

16. Isermann, B., Hendrickson, S.B., Hutley, K., Wing, M., and Weiler, H. 2001. Tissue-restricted expression of thrombomodulin in the placenta rescues thrombomodulin-deficient mice from early lethality and reveals a secondary developmental block. Development. 128:827-838.

17. Weiler-Guettler, H., Aird, W.C., Husain, M., Rayburn, H., and Rosenberg, R.D. 1996. Targeting of transgene expression to the vascular endothelium of mice by homologous recombination at the thrombomodulin locus. Circ. Res. 78:180-187.

18. Kisanuki, Y.Y., et al. 2001. Tie2-Cre transgenic mice: a new model for endothelial cell-lineage analysis in vivo. Dev. Biol. 230:230-242.

19. Taylor, F.B., Jr., Peer, G.T., Lockhart, M.S., Ferrell, G., and Esmon, C.T. 2001 . Endothelial cell protein $C$ receptor plays an important role in protein C activation in vivo. Blood. 97:1685-1688.

20. Hofmann, M.A., et al. 1999. Peripheral blood mononuclear cells isolated from patients with diabetic nephropathy show increased activation of the oxidative-stress sensitive transcription factor NF-kappaB. Diabetologia. 42:222-232.

21. Wautier, M.P., et al. 2001. Activation of NADPH oxidase by AGE links oxidant stress to altered gene expression via RAGE. Am. J. Physiol. Endocrinol. Metab. 280:E685-E694.

22. Weiler-Guettler, H., et al. 1998. A targeted point mutation in thrombomodulin generates viable mice with a prethrombotic state. J. Clin. Invest. 101:1983-1991.

23. Ravanat, C., et al. 1995. Cross-reactivity of human molecular markers for detection of prethrombotic states in various animal species. Blood Coagul. Fibrinolysis. 6:446-455.

24. Schorer, A.E. 1997. Discordant effects on eicosanoids and fibrin degradation products in two murine models of antiphospholipid antibody. Thromb. Res. 85:295-304.

25. Burstein, S.A., Boyd, C.N., and Dale, G.L. 1985. Quantitation of megakaryocytopoiesis in liquid culture by enzymatic determination of acetylcholinesterase. J. Cell. Physiol. 122:159-165.

26. Cui, J., et al. 2000. Spontaneous thrombosis in mice carrying the factor V Leiden mutation. Blood. 96:4222-4226.

27. Jalbert, L.R., et al. 1998. Inactivation of the gene for anticoagulant pro- tein $\mathrm{C}$ causes lethal perinatal consumptive coagulopathy in mice. J. Clin Invest. 102:1481-1488.

28. Chan, J.C., Cornelissen, I., Collen, D., Ploplis, V.A., and Castellino, F.J. 2000. Combined factor VII/protein C deficiency results in intrauterine coagulopathy in mice. J. Clin. Invest. 105:897-903.

29. Chan, J.C., et al. 2001. The characterization of mice with a targeted combined deficiency of protein c and factor XI. Am. J. Pathol. 158:469-479.

30. Abbott, B.D., and Buckalew, A.R. 2000. Placental defects in ARNTknockout conceptus correlate with localized decreases in VEGF-R2, Ang1, and Tie-2. Dev. Dyn. 219:526-538.

31. Goldman-Wohl, D.S., Ariel, I., Greenfield, C., Lavy, Y., and Yagel, S. 2000. Tie- 2 and angiopoietin- 2 expression at the fetal-maternal interface: a receptor ligand model for vascular remodelling. Mol. Hum. Reprod. 6:81-87.

32. Dunk, C. et al. 2000. Angiopoietin-1 and angiopoietin-2 activate trophoblast Tie-2 to promote growth and migration during placental development. Am. J. Pathol. 156:2185-2199.

33. Zhou, Y., Damsky, C.H., and Fisher, S.J. 1997. Preeclampsia is associated with failure of human cytotrophoblasts to mimic a vascular adhesion phenotype. One cause of defective endovascular invasion in this syndrome? J. Clin. Invest. 99:2152-2164.

34. Orban, P.C., Chui, D., and Marth, J.D. 1992. Tissue- and site-specific DNA recombination in transgenic mice. Proc. Natl. Acad. Sci. USA. 89:6861-6865.

35. Allaart, C.F., et al. 1993. Increased risk of venous thrombosis in carriers of hereditary protein C deficiency defect. Lancet. 341:134-138.

36. Makris, M., et al. 2000. Genetic analysis, phenotypic diagnosis, and risk of venous thrombosis in families with inherited deficiencies of protein S. Blood. 95:1935-1941.

37. Melissari, E., and Kakkar, V.V. 1989. Congenital severe protein C deficiency in adults. Br. J. Haematol. 72:222-228.

38. Daryanani, S., and Wilde, J.T. 1997. Priapism in a patient with protein C deficiency. Clin. Lab. Haematol. 19:213-214.

39. Janssen, H.L., et al. 2000. Factor V Leiden mutation, prothrombin gene mutation, and deficiencies in coagulation inhibitors associated with Budd-Chiari syndrome and portal vein thrombosis: results of a case-control study. Blood. 96:2364-2368.

40. Briffa, N.P., Wilson, I., and Clarke, D.B. 1991. Surgical treatment of pulmonary hypertension in protein C deficiency. Br. Heart J. 66:460-462.

41. Truhlar, S., and Roth, A. 1998. Pulmonary ossification in association with congenital protein C deficiency. Kans. Med. 98:18-19.

42. Andrew, M., et al. 1992. Maturation of the hemostatic system during childhood. Blood. 80:1998-2005.

43. Sagripanti, A., and Carpi, A. 1998. Natural anticoagulants, aging, and thromboembolism. Exp. Gerontol. 33:891-896.

44. Lowe, G.D., et al. 1997. Epidemiology of coagulation factors, inhibitors and activation markers: the Third Glasgow MONICA Survey. I. Illustrative reference ranges by age, sex and hormone use. Br. J. Haematol. 97:775-784.

45. Scarabin, P.Y., et al. 1996. Population correlates of coagulation factor VII. Importance of age, sex, and menopausal status as determinants of activated factor VII. Arterioscler. Thromb. Vasc. Biol. 16:1170-1176.

46. Dittman, W.A., and Majerus, P.W. 1990. Structure and function of thrombomodulin: a natural anticoagulant. Blood. 75:329-336.

47. Huang, Z.F., Higuchi, D., Lasky, N., and Broze, G.J., Jr. 1997. Tissue factor pathway inhibitor gene disruption produces intrauterine lethality in mice. Blood. 90:944-951.

48. Ishiguro, K., et al. 2000. Complete antithrombin deficiency in mice results in embryonic lethality. J. Clin. Invest. 106:873-878. 